\title{
An ultra-high-resolution IonStar strategy enhancing accuracy and precision of MS1-based proteomics and an extensive comparison with state-of-the-art SWATH-MS in large-cohort quantification
}

Xue Wang ${ }^{2,3}$, Liang Jin ${ }^{3}$, Chenqi Hu³ , Shichen Shen ${ }^{1}$, Shuo Qian², Min $\mathrm{Ma}^{2}$, Xiaoyu Zhu1, Fengzhi $\mathrm{Li}^{4}$, Jianmin Wang ${ }^{5}$, Yu Tian ${ }^{3 *}$, Jun $\mathrm{Qu}^{1,2^{*}}$

${ }^{1}$ Department of Pharmaceutical Sciences, University at Buffalo, SUNY, Buffalo, NY, United States

${ }^{2}$ Department of Cell Stress Biology, Roswell Park Comprehensive Cancer Institute, Buffalo, NY, United States

${ }^{3}$ AbbVie Bioresearch Center, Worcester, MA, United States

${ }^{4}$ Department of Pharmacology and Therapeutics, Roswell Park Comprehensive Cancer Institute, Buffalo, NY, United States

${ }^{5}$ Department of Biostatistics and Bioinformatics, Roswell Park Comprehensive Cancer Institute, Buffalo, NY 14203;

\section{*Corresponding Authors}

Yu Tian, Department of Drug Metabolism and Pharmacokinetics, AbbVie Bioresearch Center, Worcester, MA, USA. E-mail:

Jun Qu, Department of Pharmaceutical Sciences, University at Buffalo, State University of New York, 318 Kapoor Hall, Buffalo, NY 14214, USA. E-mail: junqu@buffalo.edu 


\section{Table of Content}

Fig S1

Page 4

Fig S2

Page 5

Fig S3

Page 6

Fig S4

Page 7

Fig S5

Page 8

Fig S6

Page 9

Fig S7

Page 10

Supplemental Experimental Procedure

Page 11

Supplemental Results and Discussion

Page 20

Table S1

Page 23

Table S2

Page 24

Table S3

Page 24

Table S4

Page 24

Table S5

Page 24 
Supplemental Materials

Table S6

Page 24

Table S7

Page 24

Table S8

Page 24 
Supplemental Materials

FKBA_ECOLI

LSDQEIEQTLQAFEAR

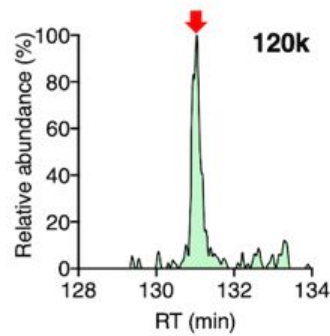

GRB2_HUMAN

VLNEECDQNWYK

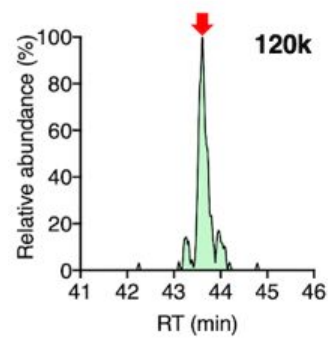

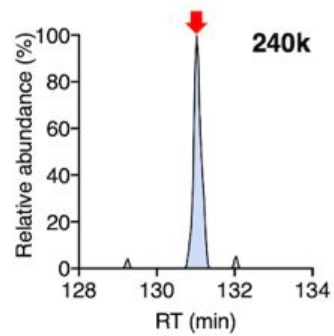

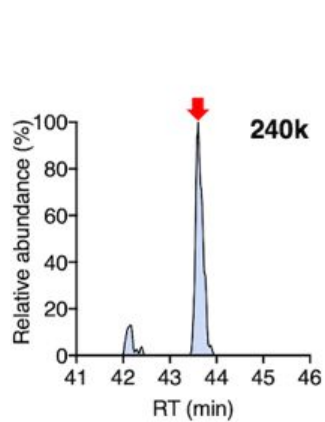

USPF_ECOLI

VISHVEEEAK
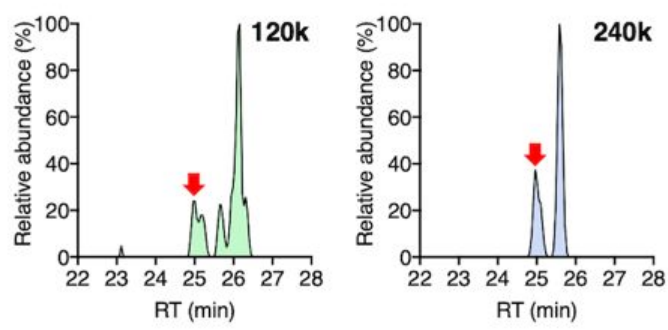

TRM1L_HUMAN

YSTPTYTGGQSESHVQSASEDTVTER
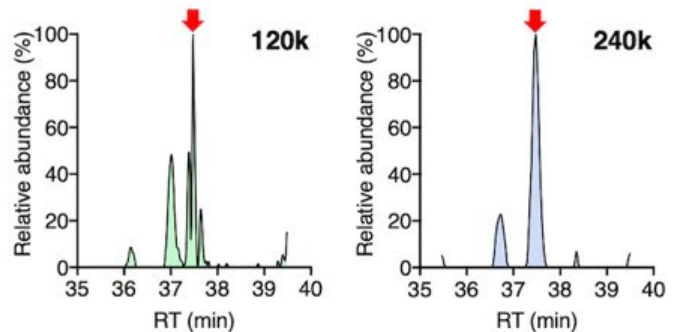

Figure S1. Improvement of sensitivity/selectivity for quantification of low-abundance peptides by ultra-high-resolution (UHR, 240k@m/z=200) MS1 measurement. 


\section{Supplemental Materials}
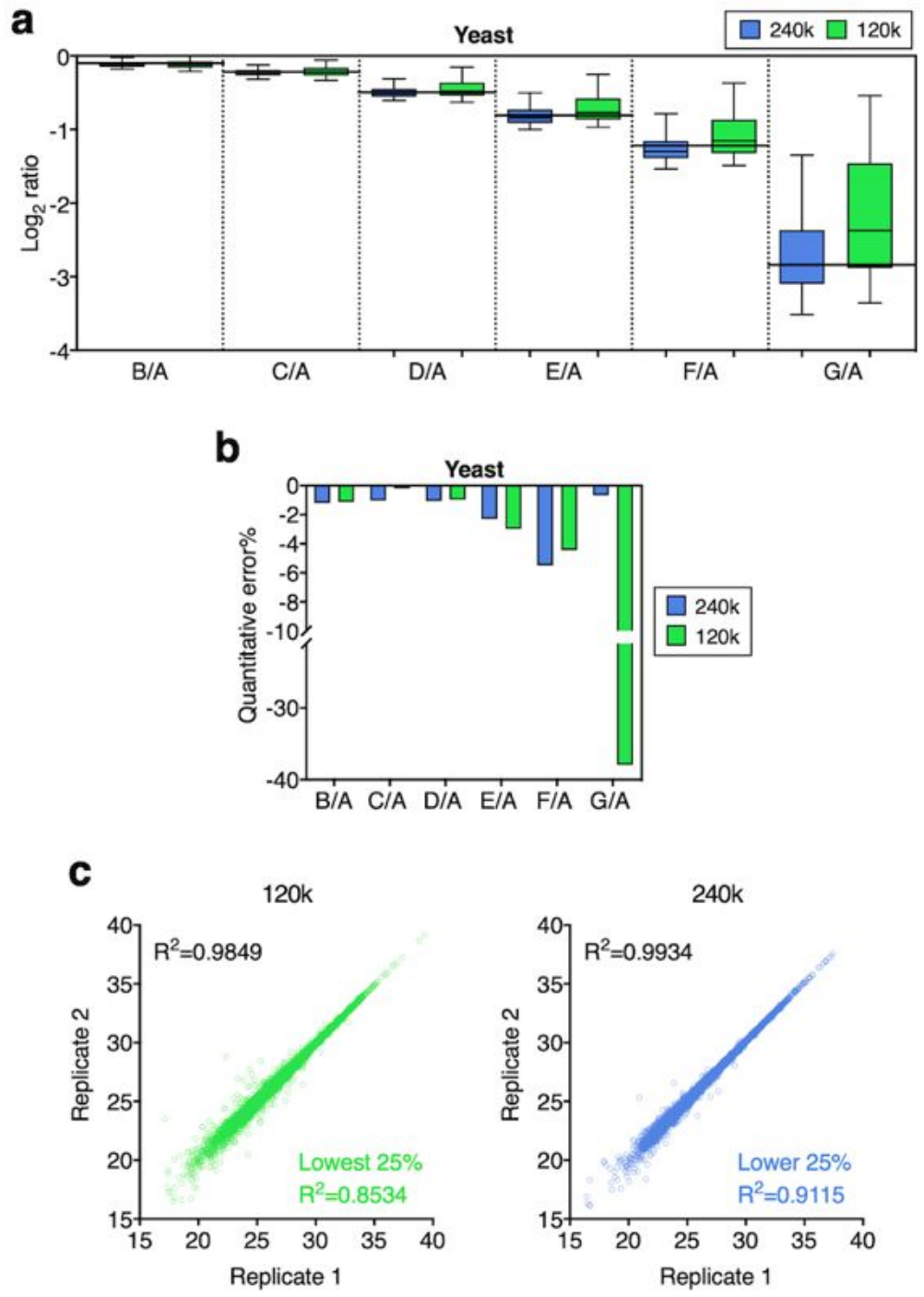

Figure S2. Evaluation of quantitative data quality by IonStar (120k) and UHR-IonStar(240k). a) The $\log _{2}$ ratios of quantified yeast proteins with $120 \mathrm{k}-\mathrm{MS}$ and 240k-MS in each two-group comparison are shown in box plots. b) The error\% of median values of quantified yeast proteins with $120 \mathrm{k}-\mathrm{MS}$ and $240 \mathrm{k}-$ MS is calculated respectively. c) The run-to-run reproducibility of quantified proteins with 120k-MS and 240k-MS is evaluated with liner regression analysis. 


\section{Supplemental Materials}

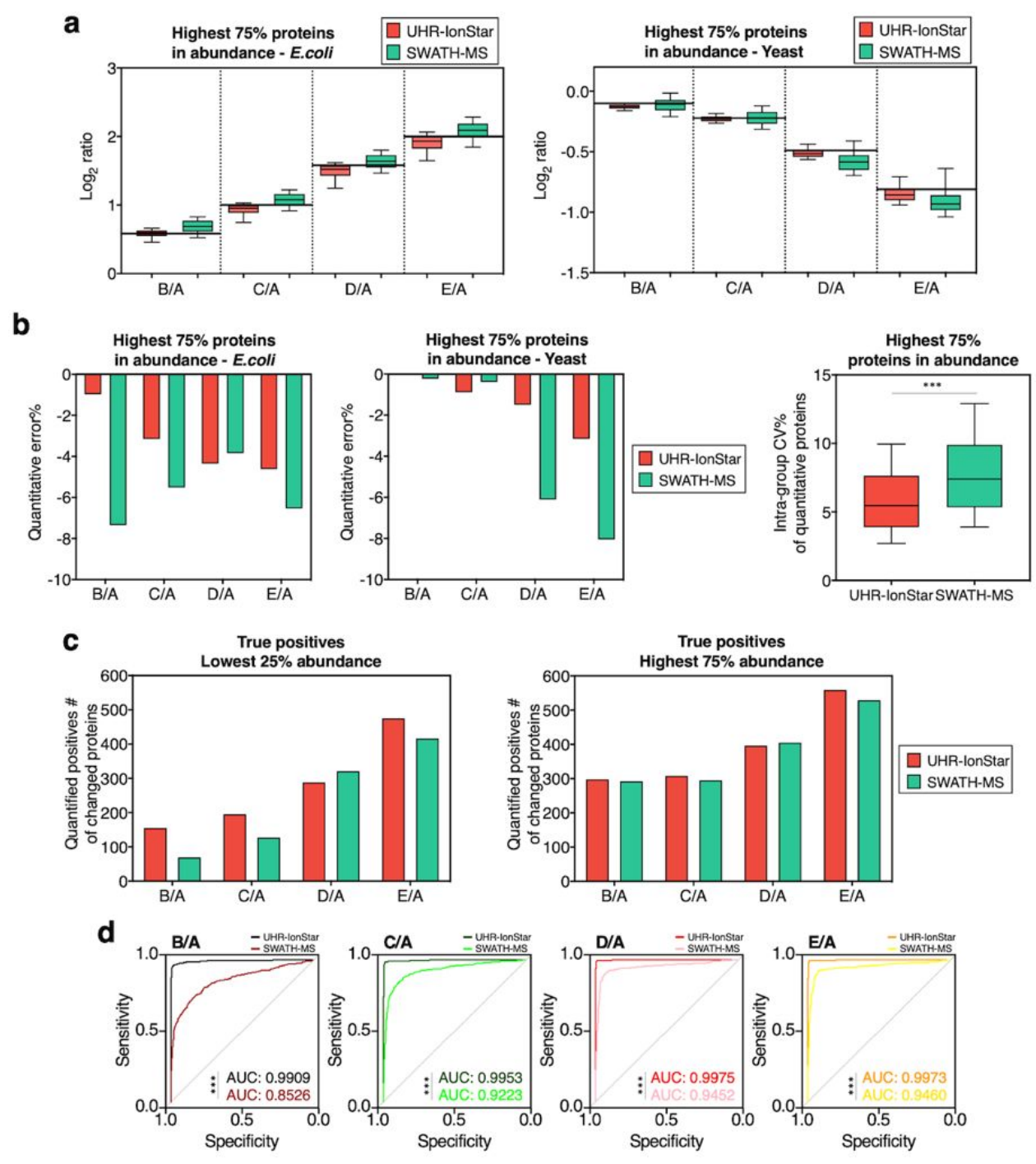

Figure S3. Evaluation of quantitative performances of UHR-IonStar and SWATH-MS in technical

replicates. a) The $\log _{2}$ ratios of quantified high-abundance E.coli proteins and yeast proteins (the highest $75 \%$ in abundance) with UHR-IonStar and SWATH-MS in each comparison. b) The error $\%$ of quantified high-abundance E.coli and yeast proteins by UHR-IonStar and SWATH-MS (left and middle); The coefficient variations of high-abundance proteins quantified by UHR-IonStar and SWATH-MS, which are shown in box plots (right). For the comparison, $p<0.001$ by one-sided Student $t$-test; c) The true positives of significantly altered low-abundance proteins and high-abundance proteins by UHR-IonStar and SWATH-MS. d) The ROCs of the sensitivity/selectivity for discovery of high-abundance, altered proteins by UHR- IonStar and SWATH-MS. ***, one-sided DeLong's test $p$-value $<0.001$. 

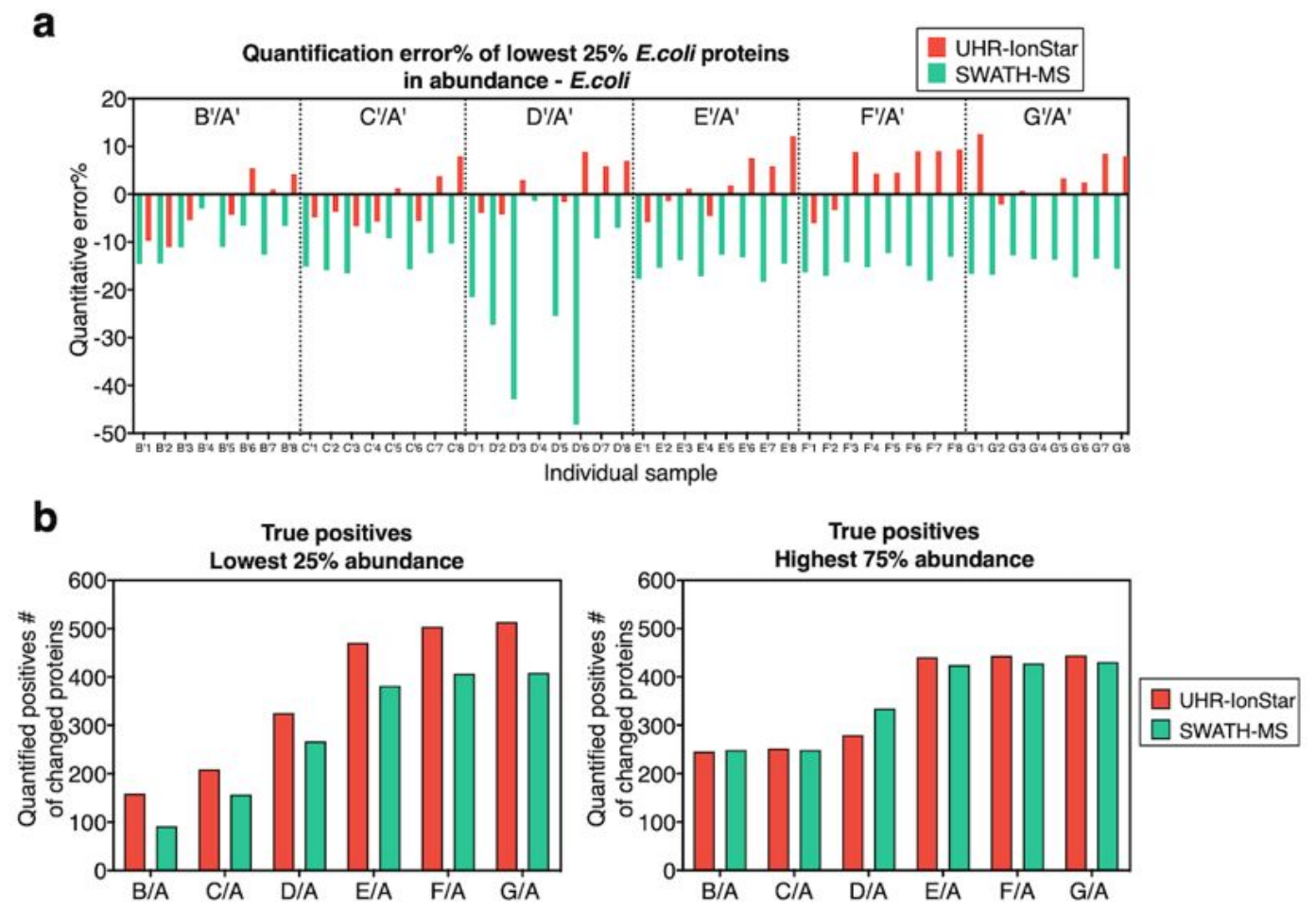

Figure S4. Comparison of UHR-IonStar and SWATH-MS in quantifying the simulated biological sample set $(\mathbf{N}=\mathbf{5 6})$. a)The median error $\%$ of the ratios of low-abundance E.coli proteins measured in each individual sample; b)The true positives discovered by UHR-IonStar and SWATH-MS in this sample set, respectively for low- and high- abundance proteins. 


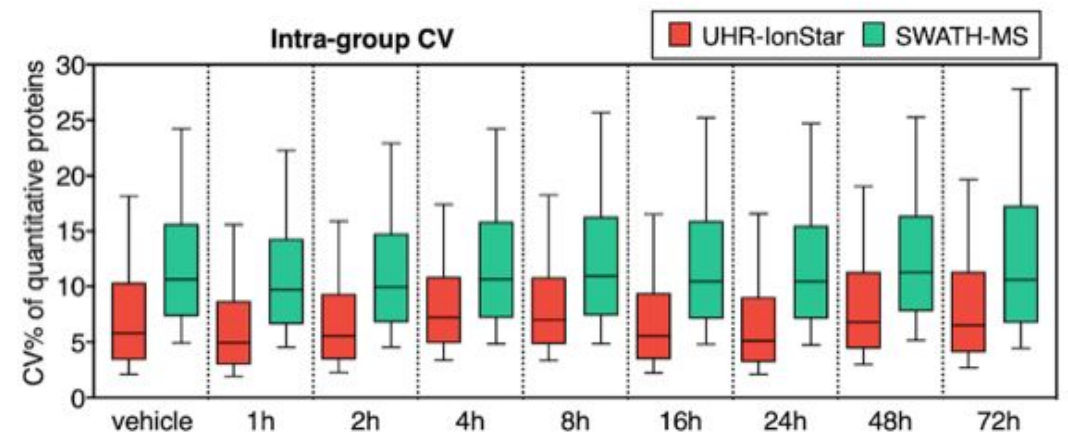

Figure S5. The intra-group CVs of quantified proteins by UHR-IonStar and SWATH-MS in each treatment group, as shown with box plots. All $p$-values are less than 0.001 from Student $t$-tests. 


\section{Supplemental Materials}

\section{a}

Highest $75 \%$ protein in abundance
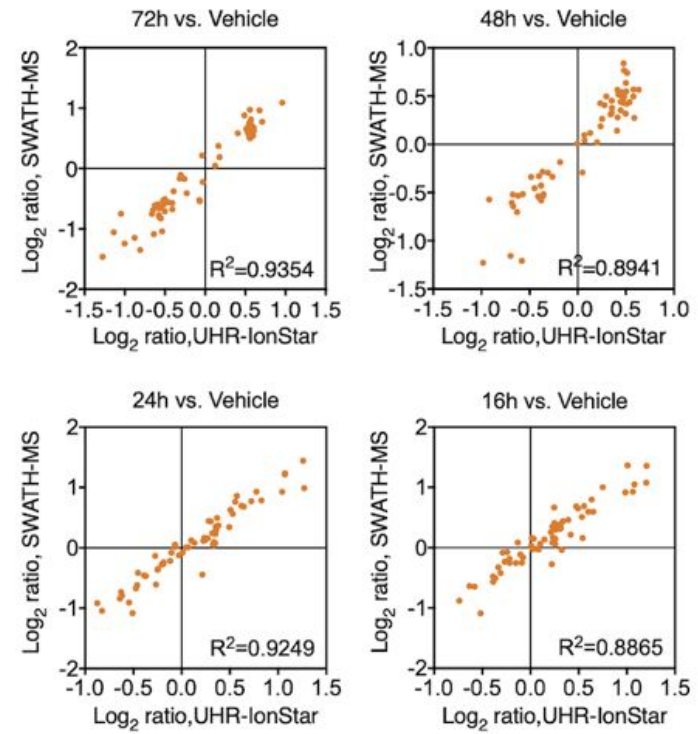

b

Lowest $\mathbf{2 5 \%}$ protein in abundance
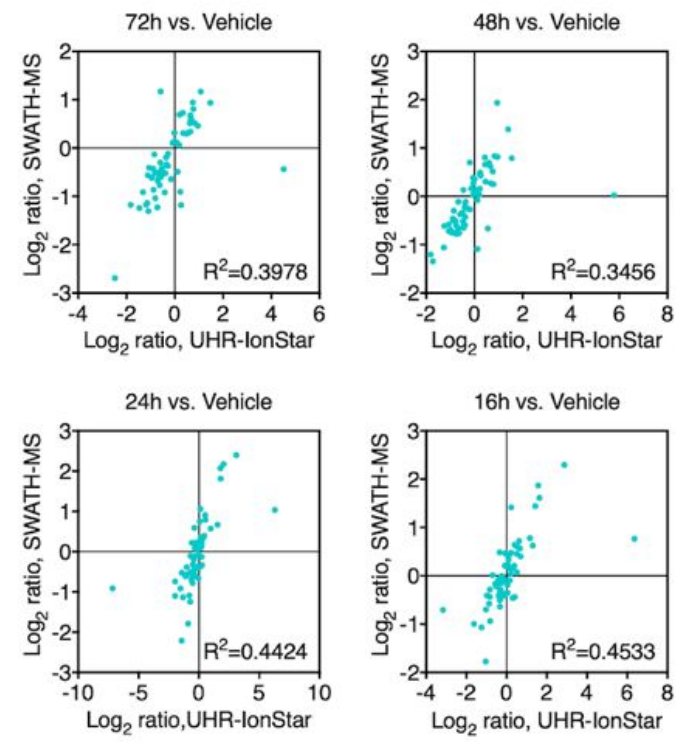

Figure S6. Correlations of the ratios of significantly-altered proteins (discovered by at least one method) in Paclitaxel-treated sample set, as measured by UHR-IonStar vs. SWATH-MS. a) The correlations of the ratios of high-abundance(the highest $75 \%$ proteins in abundance) altered proteins, measured by UHR-IonStar vs. SWATH-MS; b) The correlations of ratios of low-abundance(the lowest $25 \%$ proteins in abundance), altered proteins measured by UHR-IonStar vs. SWATH-MS. 


\section{Supplemental Materials}

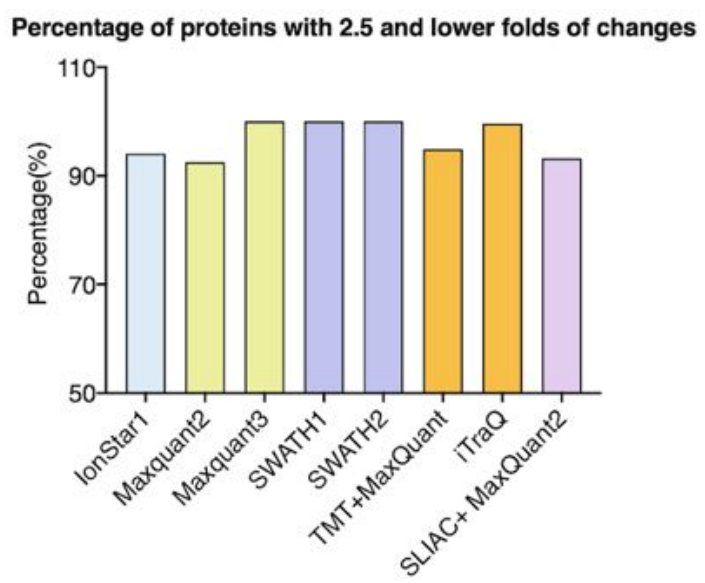

Figure S7. The percentage of discovered significantly-changed proteins with $\leq \mathbf{2 . 5}$-fold changes in proteomics literature (different methods summarized from randomly selected papers). According to extensive literature research, $90+\%$ of significantly-changed proteins discovered in proteomics projects were $\leq 2.5$ fold-change. This result underscores the importance of the capacity to accurately quantify relatively subtle changes. 


\section{Supplemental Experimental Procedure}

\section{Cell culture}

Homo sapiens colon cancer cell line SW620 was obtained from the American Type Culture Collection (ATCC, Gaithersburg, MD) and cultured in Dulbecco's minimal essential medium (DMEM) (Gibco ${ }^{\mathrm{TM}}$, NY) supplemented with $10 \%$ fetal bovine serum (FBS), $1 \%$ sodium pyruvate, $0.1 \mathrm{mg} / \mathrm{mL}$ streptomycin, $100 \mathrm{U} / \mathrm{mL}$ penicillin in humidified $37^{\circ} \mathrm{C}, 5 \% \mathrm{CO}_{2}$ incubators. Saccharomyces cerevisiae strain BY4741 was cultured in yeast extract peptone dextrose media (1\% yeast extract, $2 \%$ peptone, and $2 \%$ dextrose) at $30^{\circ} \mathrm{C}$ and $200 \mathrm{rpm}$ rotation. The media was inoculated with a starter culture with OD600 of 2.5. Cells could propagate for $\sim 12$ generations overnight to an OD600 of 2.0. E.coli strain ATCC 25922 was cultured at $37^{\circ} \mathrm{C}$ in Luria-Bertani broth medium with shaking at $250 \mathrm{rpm}$ for $\sim 15 \mathrm{~h}$. The human pancreatic cancer cell line Panc-1 (ATCC, Gaithersburg, MD) was cultured in DMEM (Corning, NY) with 10\% FBS.

\section{Sample collection and surfactant cocktail-aided extraction/precipitation/on-pellet digestion (SEPOD)}

To pellet cultured cells, Saccharomyces cerevisiae yeast cells were harvested by centrifugation at 4,000 rpm for $10 \mathrm{~min}$, E.coli cells were centrifuged at 5,000 g for $10 \mathrm{~min}$, and human cells (i.e. SW620 and Panc-1) were centrifuged at $400 \mathrm{~g}$ for $5 \mathrm{~min}$. The collected cell pellets were suspended in lysis buffer (50 mM Trisformic acid (FA), $150 \mathrm{mM}$ sodium chloride $(\mathrm{NaCl}), 0.5 \%$ sodium deoxycholate, $2 \%$ sodium dodecyl sulfate (SDS), 2\% NP-40, $\mathrm{pH} 8.0$ ) containing a cOmplete ${ }^{\mathrm{TM}}$ protease inhibitor cocktail tablet (Roche Applied Science, Indianapolis, IN) and a PhosSTOP ${ }^{\mathrm{TM}}$ phosphatase inhibitor cocktail tablet (Roche Applied Science, Indianapolis, IN). The lysate was incubated on ice for $30 \mathrm{~min}$ with vortex every $10 \mathrm{~min}$, and then homogenized 5-10 times with a Polytron homogenizer (Kinematica AG, Switzerland) for 5-10 s at 15,000 rpm with 20 s-cooling cycles. The mixture was then sonicated with a high-energy probe sonicator (Qsonica, XL-2000 or similar) for 3-5 cycles of 20 s, in order to achieve exhaustive extraction. The samples were centrifuged at $20,000 \mathrm{~g}$ for $30 \mathrm{~min}$ at $4{ }^{\circ} \mathrm{C}$, and then the supernatant was collected, the protein yield was measured with BCA protein assay (Pierce Biotechnology, Inc., Rockford, IL). The extract was stored at $80{ }^{\circ} \mathrm{C}$ until analysis. 


\section{Supplemental Materials}

For further treatment, the protein extracts were processed using a surfactant cocktail-aided extraction/precipitation/on-pellet digestion (SEPOD) procedure, which provided extensive cleanup to remove detergents and non-protein matrix components, deep protein denaturation (by both surfactants and organic-solvent precipitation) for rapid, efficient, and reproducible digestion, and thereby achieves reliable quantification. A solution containing $100 \mu \mathrm{g}$ protein was aliquoted from each sample, then diluted with $0.5 \%$ SDS to $100 \mu \mathrm{L}$ to yield a concentration of $1 \mu \mathrm{g} / \mu \mathrm{L}$. Samples were incubated with $10 \mathrm{mM}$ dithiothreitol (DTT) at $56^{\circ} \mathrm{C}$ for $30 \mathrm{~min}$ followed by $20 \mathrm{mM}$ iodoacetamide (IAM) at $37^{\circ} \mathrm{C}$ for $30 \mathrm{~min}$ in darkness. One volume $(100 \mu \mathrm{L})$ then $5 \mathrm{X}$ volume $(500 \mu \mathrm{L})$ of chilled acetone $\left(-20^{\circ} \mathrm{C}\right)$ was added to precipitate proteins, then incubated at $-20^{\circ} \mathrm{C}$ overnight. After centrifugation, $500 \mu \mathrm{L}$ methanol was added to wash the pellet. The remaining methanol was air-dried. The protein pellet was then dispersed in $80 \mu \mathrm{L}$ Tris-FA (pH 8.5) buffer in water bath sonication. For proteolytic digestion, activated trypsin was added at a ratio of 1:20 (enzyme: substrate) and incubated for $6 \mathrm{~h}$ at $37^{\circ} \mathrm{C}$. The reaction was terminated by adding $1 \mu \mathrm{L}$ FA $(1 \%$ $\mathrm{vol} / \mathrm{vol}$ final concentration). Each sample was vortexed and centrifuged at 20,000g for $30 \mathrm{~min}$ before LCMS analysis.

\section{UHR-IonStar quantitative pipeline}

\subsection{LC-MS data acquisition}

For the experimental component of UHR-IonStar, we employed a nano-RPLC system, consisting of a Dionex Ultimate 3000 nano-LC system and an Ultimate 3000 gradient micro-LC system with a WPS-3000 autosampler. Peptide separation was performed on a nano-LC column $(75-\mu \mathrm{m}$ ID $\times 50 \mathrm{~cm})$ with Pepmap $3.5 \mu \mathrm{m}$ C18 particles. An Agilent trap $(300 \mu \mathrm{m}$ ID $\times 5 \mathrm{~mm})$ packed with Zorbax $5 \mu \mathrm{m} \mathrm{C18}$ materials was employed. Nano-LC mobile phase A contains $2 \%$ acetonitrile $(\mathrm{ACN})$ with $0.1 \%$ FA, and nano-LC mobile phase B contains $88 \%$ ACN with $0.1 \%$ FA. A $4-\mu$ g peptide sample $(4 \mu \mathrm{L})$ was loaded onto the trap with $1 \% \mathrm{~B}(88 \% \mathrm{ACN}$ and $0.05 \%$ trifluoroacetic acid (TFA)) at $10 \mu \mathrm{L} / \mathrm{min}$. A selective trapping/delivery strategy, which removed most of hydrophobic/hydrophilic non-peptide matrix components, was used to ensure continuous, robust analysis of hundreds of samples. Briefly, the trapping gradients ensured efficient removal of highly hydrophilic matrix components by a 3-min wash for the trap, and then trap was switched 


\section{Supplemental Materials}

offline at 45 mins after the start of nano-flow separation phase, preventing hydrophobic components from entering the LC-MS system. After the 3-min wash for trap, a $250 \mathrm{~nL} / \mathrm{min}$ flow rate was used to back-flush the sample onto the nano-LC column for further separation. The nano-LC gradient was set as the following: 4\% B for $3 \mathrm{~min}$; 4-11 \% B for $5 \mathrm{~min} ; 11-28 \%$ B for $117 \mathrm{~min}$; 28-50 \% B for $10 \mathrm{~min}$; 50-97 \% B for $1 \mathrm{~min}$; 97\% B for 17 min. An Orbitrap Fusion Lumos Mass Spectrometer (Thermo Fisher Scientific, San Jose, CA) was employed for peptide/protein identification and quantification. Data collection was operated in a 3-s max cycle time using the data-dependent top-speed mode. The MS1 survey scan ( $m / z$ 400-1500) was at a resolution of 240,000 for UHR-IonStar or 120,000 (FWHM@m/z=200) for the previous version of IonStar with automated gain control (AGC) target of 400,000 and a maximum injection time of $50 \mathrm{~ms}$. Precursors were filtered by a quadrupole using an isolation window of $1 \mathrm{amu}$ and then fragmentized in HCD activation mode at a normalized collision energy of $35 \%$ and a dynamic exclusion duration of $45 \mathrm{~s}$. The MS2 spectra were collected at a resolution of 15,000 in the Orbitrap, with an AGC target of 50,000 and a maximum injection time of $50 \mathrm{~ms}$.

\subsection{UHR-IonStar Data processing pipeline and UHR-IonStar_v1.4}

Prior to the quantification by UHR-IonStar data processing pipeline, raw files were searched against the Uniprot-SwissProt protein database containing three species (i.e. 20,244 entries from Homo sapiens, 4,443 entries from E.coli, 6,727 entries from yeast) with MS-GF+ searching engine (released in July, 2013) for protein identification. The LC-MS files of paclitaxel-treated cells were searched against the human SwissProt database. The search parameters were set to $20-\mathrm{ppm}$ tolerance for precursor ion mass and 0.02 Da for fragment ion mass. A max of two missed cleavages were permitted for fully tryptic peptides. Carbamidomethylation of cysteine was set as a static modification, and a dynamic modification was defined as oxidation at methionine and acetylation at the $\mathrm{N}$-terminal. Other detailed search parameters can be found here ${ }^{1,2}$. Global FDR control across the entire dataset were performed with Scaffold (v.4.3.2). The identification FDR was estimated using a target-decoy search strategy that was dependent on a concatenated database of forward and reversed sequences. At least two distinct peptides were required for each identified 


\section{Supplemental Materials}

protein, and the global FDR for peptide and protein identification was set to $0.1 \%$ and $1 \%$ respectively, for the entire data set as measured by reserved database searching.

In order to take full advantage of the 240K-resolution MS1 measurement, the UHR-IonStar processing method was devised based on our previous IonStar data processing method ${ }^{1,3}$. The procedure involved using SIEVE (a commercial software from Thermofisher, who will provide access of the software to IonStar users) for chromatographic alignment and frame generation, followed by data processing modules using a GUI interface in the UHR-IonStar_v1.4 package released in this publication. The chromatographic alignment was performed with a ChromAlign algorithm modified based on the original method ${ }^{4}$ for interrun retention time (RT) adjustment. The MS1 quantitative features were generated in data-independent mode using Direct Ion Current Extraction (DICE) strategy, the defined $m / z$-RT extraction window was optimized for ultra-high-resolution MS1 measurement via using a precisely-defined, narrow window width $( \pm 5 \mathrm{ppm} \mathrm{m} / \mathrm{z}$ ) coupled with an optimized RT window width (e.g. 1 min of the aligned RT).

The UHR-IonStar web application (UHR-IonStar_v1.4, download URL: https://github.com/JunQu-

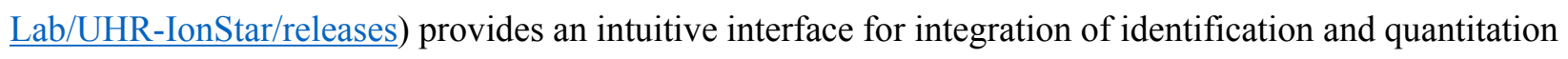
data, as well as unique approaches for post-quantification processing, data visualization and biomarker discovery. The input data include the SIEVE output SDB file (containing MS1 quantitative features), spectrum report (containing PSM information), and sample group information. The first step is frame generation, in which the frames are produced after the matching MS1 quantitative features with spectrum report by MS2 scan number, and then the redundant features (i.e. frames assigned with multiple peptide IDs) are removed. Further, the inter-sample normalization of quantitative values can be performed with quantile or total ion intensities method, at user's discretion. For quantitative consistency, UHR-IonStar performs the Principal Component-based mean-and-variance modulation to eliminate repugnant quantitative values. Finally, a model created by the sum of intensities or the Generalized Linear Mixed Model (GLMM) is used for aggregating peptide-level data to protein-level. An optional "deconvoluted proteomics" module, which is highly valuable when mixed-species proteomes are quantified (e.g. in mixed proteome benchmark or analysis of Patient-Derived Xenograft (PDX) tumor model where cancer cells are 


\section{Supplemental Materials}

of human origin while stroma cells are murine), could be implemented a step before aggregating peptide quantitative values into protein quantitative values. Using this module, only species-specific peptides are included for quantification.

The next module is "post-quantification analysis", which computes the protein ratios among groups and $p$ values produced by statistical tests. The Data Visualization step generates various types of plots: intragroup CV plot to evaluate quantitative reproducibility, intensity curve plot depicting the distribution of protein intensities in the entire dataset, inter-group correlation plot, Pearson correlation matrix plot, principal components analysis plot, and ratio distribution plot. When generating the ratio distribution plot, users can choose to rectify the protein ratios if the majority of proteins in two groups are assumed to be not significantly-different. The Changes Discovery module filters out significantly-altered proteins by the combination of two cutoff criteria: statistical test $p$-value and fold-of-change. After data filtering, data visualization using the volcano plot, up- or down-regulated protein box plot, and gene ontology analysis can be carried out. Finally, the Quantification Consistency Verification module conducts the comparison on quantitative values between the target protein and its peptides. In a reliable quantification, quantified peptides of the same protein should show consistent inter-group ratios. This step enables examination of such consistency. More details of UHR-IonStar_v1.4 can be found in the user manual ('Manual.html' in the 'UHR_IonStar_1.4' GitHub package).

\section{Comparison of the SWATH-MS and UHR-IonStar}

\subsection{Hybrid proteome benchmark sample sets}

To evaluate the performance of different quantitative strategies, two sets of concocted benchmark samples were prepared by spiking small, variable amounts of E.coli and yeast digests mimicking changed proteins (true positives) into a large, constant background of human SW620 colon cell digest representing unchanged proteins (true negatives). For both sets, a total of seven spike-in groups was included. In each sample, the percentage of yeast varied to balance the change of E.coli digest, i.e. to keep the summed proportions of the two species constant, which rendered the human protein level constant in both absolute and relative terms. The detailed design of the two benchmark sample sets is shown in Table S1. The first 


\section{Supplemental Materials}

set reflects quantification in technical replicates (Table S1a), which include the following groups (the mean percentage of E.coli proteins in total proteins): A (5\%), B (7.5\%), C (10\%), D (15\%), E (20\%), F (25\%) and G (35\%). The summed proportion of E.coli and yeast proteins is $40 \%$ in each group. Each group contains five replicates with the same proteome composition. The second set mimics large-cohort analysis of biological replicates, which includes 56 samples, each with a distinct protein composition in the following groups (the mean percentage of E.coli proteins in total proteins): A' (5\%), B' (7.5\%), C' (10\%), D' (12.5\%), E' (15\%), F' (20\%) and G' (25\%) (Table S1b). The summed proportion of E.coli and yeast proteins is $30 \%$ in each group. Each group contains eight distinct samples, where the E. coli protein levels were strategically varied so that the mean concentration in each group meet the designated value, and that E. coli protein levels in any of the B'-G' groups over A' group are statistically significant $(\mathrm{p}<0.05)$. Samples were analyzed by LC-MS in a randomized sequence in order to avoid bias. Group A' was used as control group for ratio calculation.

\subsection{Paclitaxel-treated human pancreatic cancer cell sample set}

The two methods were also evaluated by analyzing a real biological system (i.e. Paclitaxel-treated human pancreatic cancer Panc-1 cells), whose dysregulated biological processes have been very well characterized by numerous publications in the past 30 years. A time course study was performed to comprehensively capture drug-induced biological cascades and to describe the time courses of key dysregulated proteins: $1 \mathrm{~h}$, $2 \mathrm{~h}, 4 \mathrm{~h}, 8 \mathrm{~h}, 16 \mathrm{~h}, 24 \mathrm{~h}, 48 \mathrm{~h}, 72 \mathrm{~h}$, and were compared against a group of vehicle controls. Each group included four biological replicates $(\mathrm{N}=36$ in total). Both adherent cells and floating cells were harvested at each time point, and dead cells or cell debris were removed by centrifugation. The statistical significance between groups was evaluated with Student's t-test. Proteins were considered to be upregulated or downregulated with $>50 \%$ change and $p$-value $<0.05$, which was determined by the Experimental Null method ${ }^{5}$.

\section{The optimized SWATH-MS platform}

\subsection{The extensive, orthogonal $2 D$ fractionation and library building}

To build a comprehensive, project-specific spectral library of SWATH-MS, the pooled human-yeast-E.coli peptide digests (1:1:1) were fractionated using a orthogonal two-dimensional fractionation procedure which 


\section{Supplemental Materials}

coupled strong anion exchange (SAX) and high-pH reverse phase liquid chromatography (RPLC) to simplify the samples and improve the depth of identification. Firstly, SAX was performed using Pierce strong anion exchange spin column (Thermo Scientific, CA) according to the manufacturer's instructions. The spin column was conditioned using $20 \mathrm{mM}$ Tris- $\mathrm{HCl}(\mathrm{pH} 8.0)$. The $3.5 \mathrm{mg}$ tryptic peptide digests were loaded and ran through the spin column under centrifugal force of $500 \mathrm{~g}$. Flow-through and five additional fractions were collected from the spin column by washing stepwise with $20 \mathrm{mM}$ Tris- $\mathrm{HCl}$ (pH 8.0) respectively containing $0.02 \mathrm{M}, 0.1 \mathrm{M}, 0.25 \mathrm{M}, 0.5 \mathrm{M}$, and $1 \mathrm{M} \mathrm{NaCl}$. Secondly, each fraction from SAX was further fractionated using high-pH RPLC with an XBridge C18, 4.6×150 mm analytical column (Waters, Milford, MA) at a flow rate of $1 \mathrm{~mL} / \mathrm{min}$. The mobile phase consisted of $10 \mathrm{mM}$ ammonium formate (pH 9.0) in water (phase A) and 10mM ammonium formate ( $\mathrm{pH} 9.0$ ) in $90 \% \mathrm{ACN}$ (phase B). Totally 96 fractions were generated from each fraction through a 90-min gradient and then combined into 12 fractions following a fraction concatenation strategy ${ }^{6,7}$. These fractions were dried down on SpeedVac and reconstitute in 20 $\mu \mathrm{L}$ of $\mathrm{dH}_{2} \mathrm{O}$ with $2 \%$ acetonitrile and $0.1 \%$ FA for LC-MS analysis. Eventually, a total of 72 concatenated fractions (6 fractions from SAX, each further generate 12 fractions with high-pH RPLC) were collected from a single pooled sample and then subjected for LC-MS analysis and spectral library building.

\subsection{LC-MS instrumentation and data acquisition}

For SWATH-MS, the LC instrumentation included precisely metered micro-pumps, narrow bore electrode and a capillary-flow spray system enabling highly robust, reproducible separation of peptides. The metered Dionex RSLC system (ThermoFisher, San Jose) with micro liter range flow meter was used to deliver 3.1 $\mu \mathrm{L} / \mathrm{min}$ mobile phase onto a Dionex Acclaim PepMap $0.3 \times 250 \mathrm{~mm}$ C18 column packed with $2.0 \mu \mathrm{m}$ of particle size. To interface with the micro-scale flow rate, a $25-\mu \mathrm{m}$ ID hybrid electrode was fitted in the ESI probe on a Duo Spray ESI source. The narrow-bore electrode was designed for single-digit micro liter flow rate range and can be fitted with a 1/32" fitting with a Peek sleeve to hold a fused silica tubing connecting to HPLC column, through a stainless-steel nut and ferrule with a Peek sleeve. This interface setup enabled the use of a LC column with standard 1/16" fitting and standard ESI source and possessed same sensitivity as nano-LC/MS via enhanced load capacity (c.f. discussion), while significantly enhancing robustness, run- 


\section{Supplemental Materials}

to-run reproducibility. Due to the high capacity of the micro-LC column over nano-LC, an injection of 6 $\mu \mathrm{g}$ peptides were employed to achieve optimal sensitivity. The gradient was from $1 \%$ to $23.2 \%$ of buffer B in $185 \mathrm{~min}(3.1 \mu \mathrm{L} / \mathrm{min})$. Buffer A contained 2\% DMSO in water with $0.1 \%$ formic acid, and buffer B contained $2 \%$ DMSO in acetonitrile with $0.1 \%$ formic acid. The MS data acquisition was performed on a Triple TOF 6600 mass spectrometer (AB Sciex).

For DDA acquisition of spectral library building, $4 \mu \mathrm{g}$ peptides from each fraction were injected to LC-MS in the information-dependent acquisition (IDA) mode. Indexed retention time (iRT) calibration peptides (Biognosys) were spiked into each fraction before injection at 10:1 (sample: iRT, v/v). MS1 spectra were collected at the range of $400-1,500 \mathrm{~m} / \mathrm{z}$ for $250 \mathrm{~ms}$. Top 50 precursors were selected for fragmentation, the product ion MS range was from 100 to $1,800 \mathrm{amu}$ in the mode of high sensitivity. Precursors with charge 2 to 5 were selected for fragmentation, with exclusion for $30 \mathrm{~s}$ after 1 occurrence. For SWATH-MS acquisition, $8.5 \mu \mathrm{L}$ digested samples at concentration of $0.7 \mu \mathrm{g} / \mu \mathrm{L}$ of each sample was injected in the DIA mode. Full MS1 scan was performed in the range of 360 to $1,500 \mathrm{~m} / \mathrm{z}$ in positive ion mode. A total of 200 variable precursor isolation windows were determined based on the spectra data from a DIA run of $6 \mu \mathrm{g}$ pooled peptide digests using "SWATH Variable Window Calculator" provided by SCIEX. Consecutive precursor isolation windows had 1 amu overlap in $\mathrm{m} / \mathrm{z}$. The maximum accumulation time was $33 \mathrm{~ms}$ for MS1 scans and $34 \mathrm{~ms}$ for MS2 scans, resulting in a cycle time of $6.6 \mathrm{~s}$. Rolling collision energy was used to determine the collision energy for each window.

\subsection{Data processing}

DDA data of the 72 fractions from pooled hybrid proteome samples were collected for library building, which were searched by Spectronaut ${ }^{\mathrm{TM}}$ Pulsar X (version 12.0.20491.0.14754, Biognosys) against UniprotSwissprot protein database of hybrid proteome with added iRT sequences. The search parameters were set as follows: a maximum of 2 missed cleavages, carbamidomethylation of cysteines as fixed modification, oxidation of methionine and acetylation on protein $\mathrm{N}$-terminal as variable modifications, minimum peptide length was 7 and maximum peptide length was 52, cut off of peptide FDR, protein FDR and PSM FDR allset to $1 \%$, were controlled at the entire dataset level. All search results of 72 fractions were combined 


\section{Supplemental Materials}

into a final spectral library using Spectronaut. The quantitative SWATH-MS data was also analyzed by Spectronaut. In brief, MS1 and MS2 mass tolerance strategy was set as dynamic with correction factor set to 1 . Calibration mode was set to automatic. Precision iRT was enabled and local regression was selected for RT regression. Q-value cut off for both precursor and protein was set to $1 \%$. Interference correction was enabled for quantification, which required a minimum of 2 precursor-ions and 3 fragment-ions. The Qvalue percentile filter was set to $50 \%$ for data filtering. Cross runs, normalization was enabled with local normalization strategy and Q-value sparse as row selection. Quantitative protein intensity data was then $\log 2$ transferred for statistics and comparative analysis. 


\section{Supplemental Results and Discussion}

\section{Comparative evaluations of quantitative performances by UHR-IonStar and a state-of-the-art SWATH-MS method (Triple-TOF with Spectronaut)}

As discussed above, a comparative investigation of methods capable of large-cohort analysis (e.g. MS1based vs. MS2-DIA methods) has yet been conducted. Here we extensively and unbiasedly compared the UHR-IonStar with state-of-the-art SWATH-MS, on which a large-body of literature has showed much improved reproducibility and proteome coverage over the traditional MS2-DDA-based methods ${ }^{8,9}$. To this end, we employed the most advanced triple-TOF, an ideal instrument for SWATH-MS owing to its high absolute sensitivity and fast scan speed which permit selective acquisition of quantitative features via narrow, variable acquisition windows; moreover, a cutting-edge Spectronaut package was employed for SWATH data analysis. Furthermore, the method was extensively optimized to maximize its performance.

Development and optimization of a state-of-the-art SWATH-MS strategy to achieve high proteomic coverage and quantitative accuracy/precision.

First, because the sensitivity and depth of proteomics analysis by SWATH-MS are heavily dependent on the quality of spectral library, here we established and optimized a highly comprehensive, project-specific spectral library. Previously, it was shown locally-generated, project-specific libraries often achieved improved matching and quantitative performance over generic libraries from public reservoirs ${ }^{10,11}$. In this study, a library was built using pooled peptide digests. To maximize coverage, we implemented a twodimensional HPLC fractionation of digested peptides prior to LC-MS identification, by two efficient and orthogonal methods for peptide-level fractionation, strong anion exchange (SAX) and then high-pH reversed phase (high-pH-RP) ${ }^{6,12}$. Briefly, stepwise SAX elution was applied to obtain 6 fractions, and then each was separated by high-pH RPLC, fractionated into 12 concatenated fractions. This procedure produced a total of 72 fractions, which are individually subjected to capillary LC coupled to Triple-TOF MS analysis under DDA mode. Spectronaut Pulsur X was employed to perform database search and spectral library building. The spectral library (i.e. human, yeast and E. coli) contains 144,647 precursors and 121,305 


\section{Supplemental Materials}

peptides in 10,249 protein groups. Compared to the spectral library previously built from a similar mixedproteome sample set ${ }^{13}$, this library increased the peptide and protein counts respectively by 2.7 -fold and 1.5-fold, largely attributable to the efficient SAX/high-pH-RP fractionation. Such greatly enhanced comprehensiveness markedly improves depth, sensitivity and reliability of proteomic quantification.

Second, with the emphasis on precursor isolation windows, critical SWATH-MS parameters were meticulously optimized. Narrower isolation windows, especially in $\mathrm{m} / \mathrm{z}$ regions of high ion density, substantially reduce the complexity of mixed MS/MS spectra and improve selectivity, sensitivity and quantitative performance by limiting the number of precursor ions ${ }^{14}$. As conducting SWATH analysis with narrow isolation windows requires ultra-fast scan speed with high selectivity ${ }^{14}$, a state-of-the-art triple-TOF instrument for MS acquisition is advantageous. Variable window widths pre-determined by intensity distribution of precursors were employed, which were shown to markedly enhance spectral matching performance of SWATH-MS ${ }^{15,16}$. Variable window parameters were generated based on a pilot analysis of pooled samples. Other MS parameters were also optimized to achieve an optimal balance of sensitivity vs. quantitative performances for SWATH-MS, allowing enough data points measured across a typical peak. Third, LC separation was optimized to facilitate sensitive, reproducible and robust SWATH-MS analysis of large cohorts. The major technical developments included 1) Implementation of a capillary-LC instead of the nano-flow LC that is widely used for quantitative proteomics. Though nano-LC achieves higher mass sensitivity (i.e. the sensitivity expressed as the absolute amounts loaded on a column) ${ }^{17}$, it often falls short in long analytical cycles, low robustness, and compromised separation performances when compared to higher-flow LCs ${ }^{18}$, which is especially problematic for large-cohort analysis. Here the use of a capillaryflow LC approach alleviated these issues with much improved robustness, throughput and separation performances. Moreover, capillary-flow LC has much larger loading capacity over nano-LC, enabling the loading of larger number of peptides (e.g. 2-10 $\mu \mathrm{g}$, which are easily available for most proteomics projects) with excellent separation behaviors, which achieved concentration sensitivity comparable to or higher than a nano-LC/MS, a concept we demonstrated previously ${ }^{18}$. This feature was found especially effective on the triple-TOF platform. 2) We discovered that $2 \%$ DMSO as the mobile phase modifier significantly improved 


\section{Supplemental Materials}

sensitivity on the capillary-LC/Triple-TOF system. 3) An optimized micro-spray ESI system enabled highly robust and sensitive LC-MS analysis of peptides at 3.1 $\mu \mathrm{L} / \mathrm{min}$ flow rate on a small-particle capillary column. Under the optimized conditions, even for a 3-hour gradient, the typical LC peak width was only $18 \mathrm{~s}$ (FWHM) with exceptionally run-to-run reproducibility of retention times. Significantly improved quantitative performance using capillary-LC was observed over nano-LC on the Triple-TOF platform (data not shown).

As for parameters in Spectronaut data processing, we optimized Q-value cutoff for protein quantification, a critical parameter determining the quantitative data quality. Through a series of comparative evaluations, it was determined 50\% Q-value achieved an optimal balance of stringency (and therefore data quality) and proteomic coverage. 


\section{Supplemental Table}

Table S1. The designs of mix-proteome benchmark sets reflecting quantification with technical replicates and biological replicates

a.

\begin{tabular}{|c|c|c|c|c|c|c|}
\hline \multicolumn{7}{|c|}{ Theoretical ratios of technical replicates } \\
\hline & B/A & C/A & D/A & E/A & F/A & G/A \\
\hline E.coli & 1.50 & 2.00 & 3.00 & 4.00 & 5.00 & 7.00 \\
\hline Yeast & 0.93 & 0.86 & 0.71 & 0.57 & 0.43 & 0.14 \\
\hline Human & 1.00 & 1.00 & 1.00 & 1.00 & 1.00 & 1.00 \\
\hline
\end{tabular}

* The average E.coli protein content in group A is $5 \%$ of total protein mass

* Comparison between UHR-IonStar and SWATH-MS involved Group A-E.

b.

Theoretical ratios (vs. mean of group A') in the set mimicking a large cohort of biological replicates, group A'-G'

\begin{tabular}{|c|c|c|c|c|c|c|c|c|c|}
\hline & $A^{\prime} 1$ & $A^{\prime} 2$ & A'3 & $A^{\prime} 4$ & $A^{\prime} 5$ & $A^{\prime} 6$ & $A^{\prime} 7$ & $A^{\prime} 8$ & mean \\
\hline E.coli & 0.73 & 0.96 & 0.83 & 0.97 & 1.27 & 1.23 & 1.02 & 1.00 & 1.00 \\
\hline Yeast & 1.05 & 1.01 & 1.03 & 1.01 & 0.95 & 0.95 & 1.00 & 1.00 & 1.00 \\
\hline \multirow{2}{*}{ Human } & 1.00 & 1.00 & 1.00 & 1.00 & 1.00 & 1.00 & 1.00 & 1.00 & 1.00 \\
\hline & B'1 $^{\prime}$ & B'2 $^{\prime}$ & B'3 & B'4 & B'5 & B'6 & B'7 $^{\prime}$ & B' $^{\prime} 8$ & mean \\
\hline E.coli & 1.55 & 1.88 & 1.56 & 1.17 & 1.23 & 1.31 & 1.80 & 1.50 & 1.50 \\
\hline Yeast & 0.89 & 0.82 & 0.89 & 0.97 & 0.95 & 0.94 & 0.84 & 0.9 & 0.9 \\
\hline \multirow[t]{2}{*}{ Human } & 1.00 & 1.00 & 1.00 & 1.00 & 1.00 & 1.00 & 1.00 & 1.00 & 1.00 \\
\hline & $C^{\prime} 1$ & $C^{\prime} 2$ & $C^{\prime} 3$ & $C^{\prime} 4$ & $C^{\prime} 5$ & $C^{\prime} 6$ & $C^{\prime} 7$ & $C^{\prime} 8$ & mean \\
\hline E.coli & 1.88 & 1.69 & 2.03 & 1.99 & 1.84 & 2.74 & 1.83 & 2.00 & 2.00 \\
\hline Yeast & 0.82 & 0.86 & 0.79 & 0.8 & 0.83 & 0.65 & 0.83 & 0.80 & 0.80 \\
\hline \multirow[t]{2}{*}{ Human } & 1.00 & 1.00 & 1.00 & 1.00 & 1.00 & 1.00 & 1.00 & 1.00 & 1.00 \\
\hline & D'1 & D'2 & D'3 & D'4 & D'5 & D'6 & D'7 & D'8 & mean \\
\hline E.coli & 2.77 & 2.78 & 1.71 & 2.24 & 2.67 & 2.83 & 2.5 & 2.5 & 2.5 \\
\hline Yeast & 0.65 & 0.64 & 0.86 & 0.75 & 0.67 & 0.63 & 0.7 & 0.7 & 0.7 \\
\hline \multirow[t]{2}{*}{ Human } & 1.00 & 1.00 & 1.00 & 1.00 & 1.00 & 1.00 & 1.00 & 1.00 & 1.00 \\
\hline & $E^{\prime} 1$ & $E^{\prime} 2$ & E'3 & $E^{\prime} 4$ & E'5 & E'6 & $E^{\prime} 7$ & E'8 & mean \\
\hline E.coli & 3.22 & 2.49 & 3.27 & 3.58 & 2.45 & 3.21 & 2.78 & 3.00 & 3.00 \\
\hline Yeast & 0.56 & 0.7 & 0.55 & 0.48 & 0.71 & 0.56 & 0.64 & 0.60 & 0.60 \\
\hline \multirow[t]{2}{*}{ Human } & 1.00 & 1.00 & 1.00 & 1.00 & 1.00 & 1.00 & 1.00 & 1.00 & 1.00 \\
\hline & $F^{\prime} 1$ & $F^{\prime} 2$ & F'3 & $F^{\prime} 4$ & F'5 & $F^{\prime} 6$ & $F^{\prime} 7$ & $F^{\prime} 8$ & mean \\
\hline E.coli & 3.40 & 3.42 & 4.47 & 4.35 & 4.01 & 4.26 & 4.11 & 4.00 & 4.00 \\
\hline Yeast & 0.52 & 0.52 & 0.31 & 0.33 & 0.40 & 0.35 & 0.38 & 0.40 & 0.40 \\
\hline \multirow[t]{2}{*}{ Human } & 1.00 & 1.00 & 1.00 & 1.00 & 1.00 & 1.00 & 1.00 & 1.00 & 1.00 \\
\hline & $G^{\prime} 1$ & $G^{\prime} 2$ & $G^{\prime} 3$ & $G^{\prime} 4$ & G'5 & G'6 & $G^{\prime} 7$ & $G^{\prime} 8$ & mean \\
\hline E.coli & 5.46 & 4.8 & 4.92 & 4.7 & 4.95 & 5.39 & 4.78 & 5.00 & 5.00 \\
\hline Yeast & 0.11 & 0.24 & 0.22 & 0.26 & 0.21 & 0.12 & 0.24 & 0.20 & 0.20 \\
\hline Human & 1.00 & 1.00 & 1.00 & 1.00 & 1.00 & 1.00 & 1.00 & 1.00 & 1.00 \\
\hline
\end{tabular}

*The average E.coli protein content in group A is $5 \%$ of total protein mass

*The $p$-values of Student t-test comparing each of the B'-G' groups and group A are less than 0.05. 
Table S2. Quantitative proteins_UHR-IonStar_Paclitaxel-treated cells. The detailed quantitative data of proteins in paclitaxel-treated cells using UHR-IonStar.

Table S3. Quantitative proteins_SWATH-MS_Paclitaxel-treated cells. The detailed data of proteins in paclitaxel-treated cells using SWATH-MS.

Table S4. Quantitative proteins_120K_IonStar. The detailed quantitative data of proteins in the benchmark tri-proteomic system in Group A-G using IonStar.

Table S5. Quantitative proteins_UHR-Ionstar_technical replicates. The detailed quantitative data of proteins in the benchmark tri-proteomic system in Group A-G using UHR-IonStar.

Table S6. Quantitative proteins_SWATH-MS_technical replicates. The detailed quantitative data of proteins in the benchmark tri-proteomic system in Group A-E using SWATH-MS.

Table S7. Quantitative proteins_UHR-Ionstar_biological replicates. The detailed quantitative data of proteins in the benchmark tri-proteomic system in Group A'-G' using UHR-IonStar.

Table S8 Quantitative proteins_SWATH-MS_biological replicates. The detailed quantitative data of proteins in the benchmark tri-proteomic system in Group A'-G' using SWATH-MS.

Table S2, S4, S5, and S7 showed the quantitative MS intensity of each protein in log2 scale. Individual .xlsx files of each supplementary tables can be found in the supplemental materials. 


\section{Reference}

(1) Shen, X.; Shen, S.; Li, J.; etc. IonStar enables high-precision, low-missing-data proteomics quantification in large biological cohorts. Proc. Natl. Acad. Sci. U.S.A. 2018, 115, E4767-E4776.

(2) Wang, X.; Niu, J.; Li, J.; etc. Temporal Effects of Combined Birinapant and Paclitaxel on Pancreatic Cancer Cells Investigated via Large-Scale, Ion-Current-Based Quantitative Proteomics (IonStar). Mol. Cell. Proteomics 2018, 17, 655-671.

(3) Shen, X.; Shen, S.; Li, J.; etc. An IonStar Experimental Strategy for MS1 Ion Current-Based Quantification Using Ultrahigh-Field Orbitrap: Reproducible, In-Depth, and Accurate Protein Measurement in Large Cohorts. J. Proteome Res. 2017, 16, 2445-2456.

(4) Sadygov, R. G.; Maroto, F. M.; Huhmer, A. F. R. ChromAlign: A two-step algorithmic procedure for time alignment of three-dimensional LC-MS chromatographic surfaces. Anal. Chem. 2006, 78, 82078217.

(5) Shen, X.; Hu, Q.; Li, J.; etc. Experimental Null Method to Guide the Development of Technical Procedures and to Control False-Positive Discovery in Quantitative Proteomics. J. Proteome Res. 2015, 14, 4147-4157.

(6) Yang, F.; Shen, Y. F.; Camp, D. G.; etc. High-pH reversed-phase chromatography with fraction concatenation for 2D proteomic analysis. Expert Rev. Proteomics 2012, 9, 129-134.

(7) Wang, H.; Sun, S. N.; Zhang, Y.; etc. An off-line high pH reversed-phase fractionation and nanoliquid chromatography-mass spectrometry method for global proteomic profiling of cell lines. $J$. Chromatogr., B: Anal. Technol. Biomed. Life Sci. 2015, 974, 90-95.

(8) Krasny, L.; Bland, P.; Kogata, N.; etc. SWATH mass spectrometry as a tool for quantitative profiling of the matrisome. J. Proteomics 2018, 189, 11-12.

(9) Ludwig, C.; Gillet, L.; Rosenberger, G.; etc. Data-independent acquisition-based SWATH-MS for quantitative proteomics: a tutorial. Mol. Syst. Biol. 2018, 14, e8126.

(10) Govaert, E.; Van Steendam, K.; Willems, S.; etc. Comparison of fractionation proteomics for local SWATH library building. Proteomics 2017, 17, 1700052.

(11) Anjo, S. I.; Santa, C.; Manadas, B. SWATH-MS as a tool for biomarker discovery: From basic research to clinical applications. Proteomics 2017, 17, 1600278.

(12) Wang, Y. X.; Yang, F.; Gritsenko, M. A.; etc. Reversed-phase chromatography with multiple fraction concatenation strategy for proteome profiling of human MCF10A cells. Proteomics 2011, 11, 2019-2026.

(13) Navarro, P.; Kuharev, J.; Gillett, L. C.; etc. A multicenter study benchmarks software tools for labelfree proteome quantification. Nat. Biotechnol. 2016, 34, 1130-1136.

(14) Gillet, L. C.; Navarro, P.; Tate, S.; etc. Targeted data extraction of the MS/MS spectra generated by data-independent acquisition: a new concept for consistent and accurate proteome analysis. Mol. Cell. Proteomics 2012, 11, O111.016717.

(15) Zhang, Y.; Bilbao, A.; Bruderer, T.; etc. The Use of Variable Q1 Isolation Windows Improves Selectivity in LC-SWATH-MS Acquisition. J. Proteome Res. 2015, 14, 4359-4371.

(16) Schilling, B.; Gibson, B. W.; Hunter, C. L. Generation of High-Quality SWATH((R)) Acquisition Data for Label-free Quantitative Proteomics Studies Using TripleTOF((R)) Mass Spectrometers. Methods Mol. Med. 2017, 1550, 223-233.

(17) Zhang, Z.; Wu, S.; Stenoien, D. L.; etc. High-throughput proteomics. Annu. Rev. Anal. Chem. (Palo Alto, Calif.) 2014, 7, 427-454.

(18) Zhang, M.; An, B.; Qu, Y.; etc. Sensitive, High-Throughput, and Robust Trapping-Micro-LC-MS Strategy for the Quantification of Biomarkers and Antibody Biotherapeutics. Anal. Chem. 2018, 90, 1870-1880. 